\title{
Evaluating Energy Forward Dynamics Modeled as a Subordinated Hilbert-Space Linear Functional
}

\author{
Victor Alexander Okhuese ${ }^{1}$, Jane Akinyi Aduda², Joseph Mung'atu² \\ ${ }^{1}$ Department of Mathematics, Pan African University Institute for Basic Science Technology and Innovation, Nairobi, Kenya \\ ${ }^{2}$ College of Pure and Applied Sciences, Jomo Kenyatta University of Agriculture and Technology, Nairobi, Kenya \\ Email: ^alexandervictor16@yahoo.com
}

How to cite this paper: Okhuese, V.A. Aduda, J.A. and Mung'atu, J. (2020) Evaluating Energy Forward Dynamics Modeled as a Subordinated Hilbert-Space Linear Functional. Journal of Mathematical Finance, 10 , 412-430.

https://doi.org/10.4236/jmf.2020.103025

Received: June 24, 2020

Accepted: August 22, 2020

Published: August 25, 2020

Copyright $\odot 2020$ by author(s) and Scientific Research Publishing Inc. This work is licensed under the Creative Commons Attribution International License (CC BY 4.0).

http://creativecommons.org/licenses/by/4.0/

\begin{abstract}
In this study, we evaluate energy forward dynamics modeled as time-change Hilbert-space of linear functional. The energy forward is represented as an element of Hilbert-space of function. Representing energy forward and futures contracts as a time-changing stochastic process in a Hilbert-space of functions shows clearly, that an arbitrage-free forward price can be derived from the buy-and hold strategy in the energy market thereby enabling investors in the market willing to be salvage from the market uncertainties as well as Arrow-Debreu situations to execute a spot or forward contracts depending on the time and place the market becomes favorable. With a clock measuring speed of evolution or data frequency for the energy stock market, the distribution of the increments of the Lévy process with the subordinator is subordinated to the distribution of increments of the Lévy process and the results are utilized to price forward contracts of a sample electricity commodity.
\end{abstract}

\section{Keywords}

Energy, Subordination, Hilbert-Space, Jump-Diffusion, Prices, Stochastic, Futures, Forwards, Contract, Volatility, Lévy Process

\section{Introduction}

The forward pricing dynamics of an incomplete Arrow Debreu world reveals interesting challenges to speculators and one such challenge is the stochastic nature of the return process for every investment in an underlying commodity stock. In this study, we define such return process as $X(t)$ and assume a frequently changing property of time series data in discrete space. Also, the pricing 
model of the return process is assumed to be impacted by the fluctuation in the price caused either by contago or normal backwardation. Hence, the evaluation of the forward dynamics of the underlying commodity while incorporating the time-change component as an incomplete energy market is very significant for this study.

Interestingly, we adopt the completeness properties of a Banach space (a special type of Hilbert space) such that the return process $X(t)$ is defined in some normed space (i.e. complete, without hole) to enable us capture all discrete moving forward rates in the corresponding forward curves of the pricing dynamics, as such adopting similar approach by [1] and [2]. This approach is considered such that the distance between two nodes defined by the daily change price on the curve is defined in norm spaces with no gap in the sequence $X_{t}, X_{t+1}, X_{t+2}, \cdots, X_{t+n}$, where $n=1,2,3, \cdots$ with each representing a node in a forward curve of the energy forward contracts.

Furthermore, for our time-change evaluation process and according to [3], the usual subordination procedure can be used to generate a Banach space valued Lévy processes and with the dynamics from other commodities markets like power and gas we pick a motivation for this study due to the presence of strong seasonality patterns, high degree of idiosyncratic risk over different market segments and leading to spikes in the forwards contract curve. Similarly, in other to capture all forward driven prices and capture the corresponding rates, the return process will be moved from general Lévy process to a subordinated Lévy process in discrete time.

Meanwhile, the arbitrage-free forward price is derived from the buy-and-hold strategy in the underlying spot commodity and the forward price dynamics is thus implied from a given stochastic model of the spot commodity based upon similar method used by [4] and [5]. Therefore, the representation of the forward price as the conditional expected value of the spot at time of delivery is represented. As such the expectation is estimated with respect to an equivalent martingale measure $\mathrm{Q}$ which is only possible if the price of the spot commodity is specified by a semimartingale dynamics.

In literature ([4] [6] [7] and [8]), it is clear that the fundamental relationship between the spot and forward is highly delicate in energy markets and it is only fair to model the forward price dynamics directly.

\section{Preliminaries}

\subsection{Characteristics Function of a Subordinated Lévy Process}

Definition 1 A return process $\{X(t)\}_{t \geq 0}$ used in this study as Lévy process, with non-decreasing paths almost surely, are called subordinators and such processes can be thought of as random models of the evolution.

A wide class of return processes appearing in application is obtained by subordination of Lévy processes with drift. Also, a stochastic time-change to the Lévy process amounts to stochastically altering the clock on which the Lévy 
process runs.

Meanwhile, for the return process $X(t)$, the subordinated process is such that

$$
X(t)=L(\Theta(t))
$$

where the subordinator $\Theta(t)$ and $L(t)$ are increasing Lévy processes with independent and stationary increments.

According to [9] every semi-martingale $X(t)$ can be written as a time-changed Brownian motion (Lévy process), where the random time $\Theta(t)$ is a positive and increasing semi-martingale. As a result, there exist an incremental and stationary Brownian motion $(B(u), u \geq 0)$ and a random time-change $\Theta(t)$ which forms an increasing stochastic process

$$
X(t)=B(\Theta(t))
$$

Hence, every semi-martingale can also be written as a time-changed Lévy process such as

$$
X(t)=X_{t}=L(\Theta(t))
$$

The distribution of increments $\Delta L(\Theta(t))$ is said to be subordinate to the distribution of increment $\Delta L(t)$; where $\Delta(t)$ is the data frequency or a clock measuring the speed of the evolution.

Meanwhile, in developing a subordinated Lévy process with varying time, the characteristic function of a subordinated process $X(t)$ is utilized which is obtained by composition of the Laplace exponent of $\Theta(t)$ with the characteristics exponent of $L(t)$.

Suppose we let $\left(\Theta_{t}\right)_{t \geq 0}$ be a subordinator that is a Lévy process whose trajectories are increasing. Since $\Theta(t)$ is a positive random variable for all $t$ it is described by its Laplace transform rather than the Fourier transform. Let the characteristic triplet of $\Theta(t)$ be $(\Theta, \rho, \alpha)$. Then the moment generating function of $\Theta(t)$ is given by;

$$
E\left[\mathrm{e}^{u \Theta_{t}}\right]=\mathrm{e}^{t L^{*}(u)}, \forall u \leq 0
$$

where

$$
L^{*}(u)=\alpha u+\int_{0}^{\infty}\left(\mathrm{e}^{u x}-1\right) \rho(\mathrm{d} x)
$$

and $L^{*}(u)$ is the Laplace exponent of $\Theta(t)$. Since the time-changed process $X_{t}=L_{\Theta_{t}}=L\left(\Theta_{t}\right)$ is a stochastic process evaluated at a stochastic time, its characteristics function involves expectation over two sources of randomness

$$
\begin{gathered}
\Phi_{X_{t}}(u)=E\left[\mathrm{e}^{i u L_{\Theta_{t}}}\right] \\
\Phi_{X_{t}}(u)=E\left[E\left[\mathrm{e}^{i u L_{\epsilon}} \mid \Theta_{t}=\epsilon\right]\right]
\end{gathered}
$$

where the inside expectation is taken on $L_{\Theta_{t}}$, conditional on a fixed value of $\Theta_{t}=\epsilon$ and the outside expectation is on all possible value of $\Theta_{t}$. If the random time $\Theta_{t}$ is independent of $L_{t}$, the randomness due to the Lévy process can be 
integrated out using moment generating form of $L_{t}$ given by

$$
\begin{gathered}
\Phi_{L_{t}}(u)=E\left[\mathrm{e}^{i u L_{t}}\right] \\
\Phi_{L_{t}}(u)=\left[\mathrm{e}^{-i \Psi_{L}(u)}\right]
\end{gathered}
$$

and a characteristics function of $X_{t}$, that is

$$
\begin{gathered}
\Phi_{X_{t}}(u)=E\left[\mathrm{e}^{i u L_{\Theta_{t}}}\right] \\
\Phi_{X_{t}}(u)=E\left[E\left[\mathrm{e}^{i u L_{\epsilon}} \mid \Theta_{t}=\epsilon\right]\right] \\
\Phi_{X_{t}}(u)=E \mathrm{e}^{-i \Psi_{L}(u)} \\
\Phi_{X_{t}}(u)=L_{\Theta_{t}}^{*}\left(\Psi_{L}(u)\right)
\end{gathered}
$$

Under independence, the characteristics function of $X_{t}=L_{\Theta_{t}}$ is just the Laplace transform of $\Theta_{t}$ evaluated at the characteristics component of $L_{t}$.

Let $\left(L_{t}\right)_{t \geq 0}$ be a Lévy Process on $\mathbb{R}$ with characteristics exponent $\Psi(u)$ and triplet $(\sigma, v, \gamma)$ and let $\left(\Theta_{t}\right)_{t \geq 0}$ be a subordinator with Laplace exponent $L^{*}(u)$ and triplet $(\Theta, \rho, a)$. Then the subordinated process $X(t)$ defined by;

$$
X(t)=L(\Theta(t)),
$$

is also a subordinated Lévy process with characteristics function

$$
E\left[\mathrm{e}^{i u X_{t}}\right]=\mathrm{e}^{t L^{*}(\Psi(u))} .
$$

Therefore, in defining a parametric Lévy process is to obtain an Lévy process by subordinating a Brownian motion with an independent increasing Lévy process. However, for a continuous time change process, we it is necessary to evaluate the stochastic time integral of the subordinated Lévy process linear in each argument.

\subsection{Stochastic Integrals of a Subordinated Lévy Process in Hilbert Space}

Since the time-changed return processes $X_{t}$ and $Y_{t}$ are independent subordinated Lévy processes evaluated at a stochastic time, and using the approach in [10], we derive some general results on the stochastic integral represented as linear functional in a subordinated Hilbert space. Suppose we define a subordinated stochastic integral $Y$ in the form

$$
Y(t)=\int_{0}^{t} \Psi(s) \mathrm{d} L(s)
$$

for a Lévy process $L(t)$ with values in a separable Hilbert space $U$ and an integral stochastic process $\Psi: \mathbb{R}_{+} \times U \rightarrow H$, and considering the stochastic partial differential equation

$$
\mathrm{d} X(t)=(A X(t)+\alpha(t)) \mathrm{d} t+\sigma(t) \mathrm{d} L(t), t \in \mathbb{R}
$$

on the interval $\mathbb{R}=[0, T]$ with $H$ being a separable Hilbert spaces equipped with some boundary conditions where 
- $A: H \rightarrow H$ is a first order differential operator

- $\alpha: \mathbb{R} \rightarrow H$ is a mapping with Brochner-integrable trajectories

- $L: \mathbb{R} \rightarrow H$ is a square-integrable, $H$-valued stochastic process with covariance operator $Q \in L_{1}^{+}$.

- $\sigma: \mathbb{R} \rightarrow L\left(Q^{1 / 2}(H), H\right)$ is an operator-valued process.

Suppose for convenience, we are only interested in one-dimensional martingales, i.e., we are interested in

$$
Y(t)=\Upsilon(Y(t)),
$$

where $\Upsilon$ is a continuous linear functional on the state space $H$ of $Y$. Therefore, by replacing $Y(t)$ with $X(t)$ assuming the characteristic of the subordinated process we have;

$$
X(t)=\int_{0}^{t}(\Upsilon \circ \Psi(s)) \mathrm{d} L(s) .
$$

Suppose we take $U$ as finite dimensional, and some standard (real-valued) Brownian motion $\{B(t)\}_{t \geq 0}$ and Itô integral stochastic process $\sigma$ such that

$$
X(t)=\int_{0}^{t} \sigma(s) \mathrm{d} B(s)
$$

This leads us to Theorem 1 which shows that a similar representation holds if $U$ is any separable Hilbert space.

Theorem 1. Let $n \in \mathbb{N}$ and $H, U$ be separable Hilbert spaces. Let $L$ be a square integrable and mean zero $U$-valued Wiener process with coveriance $Q \in L_{1}^{+}$. Assume that $\operatorname{Dimran}(Q) \geq n$ and $Q$ is positive definite. Let $\Psi \in \aleph_{L}^{2}$, $\Upsilon \in L\left(H, \mathbb{R}^{n}\right)$ and define

$$
X(t)=\Upsilon\left(\int_{0}^{t} \Psi(s) \mathrm{d} L(s)\right) .
$$

Then there is an $n$-dimensional standard Brownian motion $B$ such that

$$
X(t)=\int_{0}^{t} \sigma(s) \mathrm{d} B(s),
$$

where $\sigma(s)=\left(\Upsilon \Psi(s) Q \Psi(s)^{*} \Upsilon^{*}\right)^{1 / 2} \in \aleph_{B}^{2}\left(\mathbb{R}^{*}\right)$. If $\sigma(s)$ is invertible in $\mathbb{R}^{n \times n}$ for $\lambda \otimes P$-almost any $s \in \mathbb{R}$, then $\sigma^{-1} \in \aleph_{X}^{2}\left(\mathbb{R}^{n}\right)$ and

$$
B(t)=\int_{0}^{t}(\sigma(s))^{-1} \mathrm{~d} X(s) .
$$

Proof. Let $\Psi$ be an elementary random variable such that there exist $n \in \mathbb{N}$, $0 \leq a_{j} \leq b_{j}<\infty$, and $F_{a_{j}}$-measurable square integrable random variables $Y_{j}$ and $\psi_{j} \in L(U, H)$ such that

$$
\Psi=\sum_{j=1}^{n} Y_{j} 1_{\left[a_{j}, b_{j}\right] \psi_{j}} .
$$

By definition of $L$, we have $L(t)=W(\Theta(t))$ for a $U$-valued Wiener process $W$ with martingale covariance $Q^{W}$ and a subordinator $\Theta$. Let $\Gamma$ be the isometric embedding given in Lemma (1). Note that $\Gamma(\Upsilon \Psi=\Upsilon \Gamma(\Psi))$ because this holds if $\Psi$ is elementary. From Lemma (1) and Theorem (1) we have; 


$$
\begin{aligned}
\Upsilon\left(\int_{0}^{t} \Psi(s) \mathrm{d} L(s)\right) & =\int_{0}^{t} \Upsilon \Psi(s) \mathrm{d} L(s) \\
& =\int_{0}^{\Theta(t)} \Upsilon \Gamma(\Psi)(s) \mathrm{d} W(s) \\
& =\int_{0}^{\Theta(t)} \sigma_{1}(s) \mathrm{d} B(s)
\end{aligned}
$$

where $B$ is a standard Brownian motion on $\mathbb{R}^{n}$ and

$$
\sigma_{1}(t)=\Gamma\left(\left(\Upsilon \Psi Q^{W} \Psi^{*} \Upsilon^{*}\right)^{1 / 2}\right)(t)
$$

Applying Lemma (1) again gives

$$
\Upsilon\left(\int_{0}^{t} \Psi(s) \mathrm{d} L(s)\right)=\int_{0}^{t} \sigma(s) \mathrm{d} N(s)
$$

where $N(t)=B(\Theta(t)), \quad t \in \mathbb{R}_{+}$and

$$
\sigma(t)=\left(\Upsilon \Psi(t) Q^{W} \Psi(t)^{*} \Upsilon^{*}\right)
$$

here, $N$ is an $n$-dimensional Lévy process with the desired properties and the martingale covariance $Q^{L}$ of $L$ and $Q^{W}$ of $W$ coincide based on the above theorem ([10]).

Based on results in [3] we conclude the construction of the stochastic integral based on Equation (10);

$$
X(t)=\int_{0}^{t} \Gamma(s) \mathrm{d} L(s)
$$

where $L \in M^{2}(U)$ and $\Gamma(s)=\Upsilon \circ \Psi(s)$ are operators from $U$ to another Hilbert space $H$.

However, in order to express the subordination in terms of standard Brownian motion, we make the following definition:

Definition 2. A subordinated Brownian moion $L$ with values in some Hilbert space $U$ is a Lévy process such that there is a $U$-valued Brownian motion $B$ and a subordinator $\Theta$ which is independent of $B$ such that

$$
L(t)=B(\Theta(t)) .
$$

Subordinated Brownian motion $L, N$ are of same type if there are Brownian motions $B_{L}, W_{N}$ and subordinators $\Theta_{L}, \Theta_{N}$ such that $B_{N}, \Theta_{N}$ are independent, $B_{L}, \Theta_{L}$ are independent, $\Theta_{L}, \Theta_{N}$ have the same law and for any $t \in \mathbb{R}_{+}$. Subordinated Brownian motions have some similarities with Brownian motion if the subordinator has finite first moment. In particular, the set of integrands can be compared easily. First, we recall the notion of time-changed filtrations:

Definition 3. A time-change is a right-continuous increasing family $(\Theta(t))_{t \in \mathbb{R}}$ of stopping times with respect to some right-continuous filtration $\left(F_{t}\right)_{t \in \mathbb{R}_{+}}$. The time changed filtration is the filtration given by

$$
F_{t}^{\Theta}=F_{\Theta(t)}=\bigcap_{s>t} F_{\Theta(s)} t \in \mathbb{R}_{+} .
$$

Let $X$ be an $F$-adapted stochastic process and assume that the time-change is 
finite valued. The time-changed process by $X^{\Theta}(t)=X(\Theta(t)), t \in \mathbb{R}_{+}$. Therefore, adding the following results on stochastic integration with respect to subordinated Brownian motions.

Lemma 1. Let $B$ be a mean-zero Brownian motion with values in a separable Hilbert space $U$ relative to some filtration $\left(F_{t}\right)_{t \in \mathbb{R}_{+}}, H$ be another separable Hilbert space, $\psi \in \aleph_{L}^{2}(G, H), \Theta$ be a non-zero subordinator with finite moment such that $\Theta(t)$ is a stopping time for each $t \in \mathbb{R}_{+}$. Let $\left(G_{t}\right)_{t \in \mathbb{R}_{+}}$be the time-changed filtration given by $G_{t}=F_{\Theta(t)}$ and $L(t)=B(\Theta(t)), \quad t \in \mathbb{R}_{+}$. Then $L$ is a $U$-valued square integrable Lévy process and there is an isometric embedding $\Gamma: \aleph_{L}^{2}(G, H) \rightarrow \aleph_{B}^{2}(F, H)$ such that

$$
\int_{0}^{t} \psi(s) \mathrm{d} L(s)=\int_{0}^{\Theta(t)} \Gamma(\psi)(s) \mathrm{d} B(s), P \text {-almost surely }
$$

where the left stochastic integral is with respect to the filtration $\left(G_{t}\right)_{t \in \mathbb{R}_{+}}$, the right stochastic integral is with respect to the filtration $\left(F_{t}\right)_{t \in \mathbb{R}_{+}}$.

Proof: See proof in [7].

Therefore, we can see from the above construction that Equation (14) holds for elementary integrands, and thus for all integrands by a density argument in the Hilbert space. We can use results from Lemma (1) to derive a link between functionals of the infinite-dimensional stochastic integral and finite dimensional versions of it.

\subsection{Evaluating Energy Forward Dynamics as a Subordinated Hilbert Space}

In this section, we use the properties of Hilbert space to represent the forward and futures prices in energy markets as an element of Hilbert space of functions. Motivated by results in [10], it is observed that the various relevant forwards and futures contracts traded in energy markets, which deliver the underlying over a period rather than at a fixed time in the future, can be understood as a bounded operator on a suitable Hilbert space.

We begin by stating the following relevant assumption from the Filipovic space [11] which supports the Hilbert space appropriate for our considerations.

H1: The functions $h \in H$ are continuous and the pointwise evaluation $\mathcal{J}_{\tau}(h)=h(\tau)$ is a continuous linear functional on $H$, for all $\tau \in R_{+}$.

$\mathrm{H} 2$ : The semigroup $\left\{S(t) \mid t \in \mathbb{R}_{+}\right\}$is strongly continuous in $H$ with infinitesimal generator denoted by $A$.

Therefore, we use the previously defined class of Hilbert space which follows from the Filipovic space satisfying ( $\mathrm{H} 1)-(\mathrm{H} 2)$ and which are coherent with economical reasoning about the forward curve $\tau \leftrightarrow h(\tau)$.

Since in practice the forward curve is obtained by smoothing data points using smooth fitting methods it is reasonable to assume;

$$
\int_{0}^{\infty} h^{\prime}(\tau)^{2} \mathrm{~d} \tau<\infty
$$

defined on $H_{w}$ of absolutely continuous functions $h: \mathbb{R}_{+} \rightarrow \mathbb{R}$. Moreover, the 
curve flattens for large time to maturity $\tau$. There is no reason to believe that the forward rate for an instantaneous loan that begins in 10 years differs much from one which one day later. We take this into account by penalizing irregularities of $h(\tau)$ for large $\tau$ by some increasing weighing function $w(\tau) \geq 1$, that $\int_{0}^{\infty} w(\tau) h^{\prime}(\tau)^{2} \mathrm{~d} \tau$ for a given continuous and increasing weight function $w: \mathbb{R}_{+} \rightarrow[1, \infty)$ with $w(0)=1$. The norm of $H_{w}$ is $\|g\|_{w}^{2}=\langle g, g\rangle$ for the inner product

$$
\langle f, h\rangle=f(0) h(0)+\int_{0}^{\infty} w(\tau) h^{\prime}(\tau) f^{\prime}(\tau) \mathrm{d} \tau .
$$

here $f, h \in H_{w}$. We assume that $\int_{0}^{\infty} w^{-1}(\tau) \mathrm{d} \tau<\infty$. The typical choice of weight function is that of an exponential function $w(\tau)=\mathrm{e}^{(\tilde{w} \tau)}$ for a constant $w>0$, in which case the integrability condition on the inverse of $w$ is trivially satisfied. From [11], we know that $H_{w}$ is a separable Hilbert space. As we shall see, one can realize energy forward prices as linear operators on $H_{w}$ and in fact interpret energy forward prices as stochastic processes with values in this space.

A simple motivation for the appropriate choice of $H_{w}$ is the classical model for the dynamics of energy spot prices in the so-called Schwartz dynamics [12].

Here, the spot price $S(t)$ at time $t \geq 0$ is given by

$$
S(t)=\exp (X(t))
$$

for a standardized subordinated return process $X(t)$ defined in an Ornstein-Uhlenbeck (OU) process

$$
\mathrm{d} X(t)=\mu \mathrm{d} t+\sigma \mathrm{d} L(t)
$$

with a corresponding risk-neutral process given by

$$
\mathrm{d} X(t)=\alpha(\mu-X(t)) \mathrm{d} t+\sigma \mathrm{d} L(t),
$$

driven by a Lévy process $L(t)$. We assume that $L(1)$ has exponential moments, $\rho>0, \mu$ are constants, and $\ln S(0)=X(0)=\tau \in \mathbb{R}$. It is simple to see that $\tau \mapsto f(t, \tau)$ is continuously differentiable for every $t$, and

$$
\frac{\partial f}{\partial \tau}(t, \tau)=f(t, \tau)\left(\alpha \mathrm{e}^{-\alpha \tau}(\mu-X(t))+\phi\left(\mathrm{e}^{-\alpha \tau}\right)\right) .
$$

From [12], we get the forward price $f(t, T)$ at time $t \geq 0$, for a contract delivering at time $T \geq t$, is given;

$$
f(t, T)=\exp \left(\mathrm{e}^{-\alpha(T-t)} X(t)+\mu\left(1-\mathrm{e}^{-\alpha(T-t)}\right)+\int_{0}^{T-t} \phi\left(\mathrm{e}^{-\alpha s}\right) \mathrm{d} s\right)
$$

with $\phi$ being the logarithm of the moment generating function of $L(1)$. Suppose we model the spot price directly under the pricing measure $Q$. Letting $\tau=T-t \geq 0$, we find (by slightly abusing the notation)

$$
f(t, \tau)=\exp \left(\mathrm{e}^{-\alpha(\tau)} X(t)+\mu\left(1-\mathrm{e}^{-\alpha(\tau)}\right)+\int_{0}^{\tau} \phi\left(\mathrm{e}^{-\alpha s}\right) \mathrm{d} s\right) .
$$

Assuming that the weight function $w$ is such that

$$
w(\tau) \mathrm{e}^{-2 \alpha \tau} \in L^{1}\left(\mathbb{R}_{+}\right) .
$$


Then it follows that $\int_{0}^{\infty}|\phi(\exp (-\alpha \tau))| \mathrm{d} s<\infty$ from the Cauchy-Schwartz inequality and the assumption $\int_{0}^{\infty} w^{-1}(\tau) \mathrm{d} \tau<\infty$. Hence, $f$ is uniformly bounded in $\tau$ since

$$
|f(t, \tau)| \leq \exp \left(X(t)+\mu+\int_{0}^{\infty}\left|\phi\left(\mathrm{e}^{-w s}\right)\right| \mathrm{d} s\right)
$$

But then

$$
\begin{aligned}
\|f(t, .)\|_{w}^{2} & =|\exp (X(t))|^{2}+\int_{0}^{\infty} w(\tau) f^{2}(t, \tau)\left(\alpha \mathrm{e}^{-\alpha \tau}(\mu-X(t))+\phi\left(\mathrm{e}^{-\alpha x}\right)\right)^{2} \mathrm{~d} x \\
& \leq c \mathrm{e}^{2 X(t)}\left(1+\int_{0}^{\infty} w(\tau) \mathrm{e}^{-2 \alpha \tau} \mathrm{d} \tau+\int_{0}^{\infty} w(\tau) \phi^{2}\left(\mathrm{e}^{-\alpha \tau}\right) \mathrm{d} \tau\right),
\end{aligned}
$$

which shows that $f(t,.) \in H_{w}$. If $L$ is a driftless Lévy process, the exponential moment condition on $L(1)$ yields that $\phi(\tau)$ has the representation

$$
\phi(\tau)=\frac{1}{2} \sigma^{2} \tau^{2}+\int_{\mathbb{R}}\left\{\mathrm{e}^{\tau z}-1-\tau z\right\} v(\mathrm{~d} z)
$$

for a constant $\sigma \geq 0$ and Lévy measure $v(\mathrm{~d} z)$. But by the monotone convergence theorem and L'Hopital's rule, we find that

$$
\lim _{\tau \rightarrow 0} \frac{1}{\tau^{2}} \int_{\mathbb{R}}\left\{\mathrm{e}^{\tau z}-1-\tau z\right\} v(\mathrm{~d} z)=\frac{1}{2} \int_{\mathbb{R}} z^{2} v(\mathrm{~d} z),
$$

such that Equation (18) becomes

$$
\phi(\tau)=\frac{\tau^{2}}{2}\left(\sigma^{2}+\int_{\mathbb{R}} z^{2} v(\mathrm{~d} z)\right)
$$

and therefore $\phi(\tau) \sim \tau^{2}$ when $\tau$ is small. Thus, a sufficient condition for $f(t,.) \in H_{w}$ is $w(\tau) \mathrm{e}^{(-2 \alpha \tau)} \in L^{1}\left(\mathbb{R}_{+}, \mathbb{R}\right)$.

Therefore, we now represent the realization in $H_{w}$ of general energy forward and futures contracts with a delivery period. Suppose that $F\left(t, T_{1}, T_{2}\right)$ is the forward price at time $t$ of a contract on energy delivering over the time interval $\left[T_{1}, T_{2}\right]$, where $0 \leq t \leq T_{1}<T_{2}$ [12]. Then we can express this price as

$$
F\left(t, T_{1}, T_{2}\right)=\int_{T_{1}}^{T_{2}} \tilde{w}\left(T ; T_{1}, T_{2}\right) f(t, T) \mathrm{d} T,
$$

where $f(t, T), t \leq T$ is the forward price for a contract "delivering energy" at the fixed time $T$, and $\tilde{w}\left(T ; T_{1}, T_{2}\right)$ is a deterministic weight function defined by;

$$
\tilde{w}\left(T ;, T_{1}, T_{2}\right)=\frac{1}{T_{2}-T_{1}}
$$

for the forward-style contracts and

$$
\tilde{w}\left(T ; T_{1}, T_{2}\right)=\frac{\mathrm{e}^{-r T}}{\int_{T_{1}}^{T_{2}} \mathrm{e}^{-r s} \mathrm{~d} s}
$$

for the futures style.

Here $r>0$ is the risk-free interest rate which we suppose to be constant. In the energy market on NYMEX, say, WTI oil is delivered physically at a location over a given delivery period like month or quarter. We will therefore have the same expression (19) for the oil forward prices as in the case of energy forwards. 
Meanwhile, futures on WTI oil index deliver the money-equivalent from the aggregate index value over a specified period. Hence, the futures can be expressed as

$$
F\left(t, T_{1}, T_{2}\right)=\int_{T_{1}}^{T_{2}} f(t, T) \mathrm{d} T,
$$

where $f(t, T)$ is the futures price of a contract that delivers the corresponding index at the fixed delivery time $T \geq t$, i.e., the weight of the futures contracts can be expressed by (19) with

$$
\tilde{w}\left(T ; T_{1}, T_{2}\right)=1
$$

as the weight function. Aiming at a so-called Museila representation of $F\left(t, T_{1}, T_{2}\right)$ in (19). Define $\tau=T_{1}-t$ as the time until start of delivery of the swap and $\ell=T_{2}-T_{1}>0$ as the length of delivery of the swap. With the notation $g(t, y)=f(t, t+y)$, we write

$$
G_{\ell}^{w}=F(t, t+\tau, t+\tau+\ell)=\int_{\tau}^{\tau+\ell} w_{\ell}(t, \tau, y) g(t, y) \mathrm{d} y
$$

for the weight function $w_{\ell}(t, \tau, y)$ defined by

$$
w_{\ell}(t, \tau, y)=\tilde{w}(t+y ; t+\tau, t+\tau+\ell),
$$

where $y \in[\tau, \tau+\ell], \tau \geq 0$ and $t \geq 0$. Referring to the different cases of the weight function $\tilde{w}$, we find that $w_{\ell}(t, \tau, y)=1$ for a forward contract and $w_{\ell}(t, \tau, y)=1 / \ell$ for the forward-style oil swap. Slightly more interesting are the future-style energy swaps, yielding

$$
w_{\ell}(t, \tau, y)=\frac{r}{1-\mathrm{e}^{-r \ell}} \mathrm{e}^{-r(y-\tau)} .
$$

here, we use the future-style contract as defined in Equation (21). Note that all these cases result in a weight function $w_{\ell}$ which is independent of time. Furthermore, the only case that depend on $\tau=T-t$ and $y$ is given in Equation (25), which becomes in fact stationary in the sense that $w_{\ell}$ depends on $y-\tau$.

However, when the asset price is a spot commodity, the solution for forwards price $F(S, t, T)$ (under deterministic interest rates, futures prices equal forward prices) is given by;

$$
F(S, t, T)=S \mathrm{e}^{(r-X(t)) \tau},
$$

where $\tau=T-t$ is the time to expiration of the contract, and with $L(t)=\ln S(t)$ and hence

$$
S(t)=\mathrm{e}^{L(t)}
$$

where $L(t)$ is defined by the geometric Lévy process given by;

$$
L(t)=L(0) \mathrm{e}^{W}
$$

and

$$
\begin{aligned}
W= & \left(\alpha-\frac{1}{2} \sigma^{2}\right) t+\sigma \mathrm{d} B(t)+\int_{0}^{t} \int_{|z|<R}\{\ln (1+\gamma(s, z))-\gamma(s, z)\} v(\mathrm{~d} z) \mathrm{d} s \\
& +\int_{0}^{t} \int_{\mathbb{R}} \ln (1+\gamma(s, z)) \tilde{N}(\mathrm{~d} s, \mathrm{~d} z)
\end{aligned}
$$


For a Poisson process, $\gamma(s, z)>-1$ measures the sensitivity of the process $L(t)$ and at $R=\infty$

$$
\int_{0}^{\infty} N(\mathrm{~d} t, \mathrm{~d} z)=1
$$

and we have

$$
\begin{aligned}
W & =\left(\alpha-\frac{1}{2} \sigma^{2}\right) t+\sigma B(t)+\int_{0}^{t} \ln (1+\gamma(s, z)) \mathrm{d} t \\
& =\left(\alpha-\frac{1}{2} \sigma^{2}\right) t+\sigma B(t)+\ln (1+\gamma(s, z)) t
\end{aligned}
$$

Suppose $1+\gamma(s, z)>0$ then $\gamma>-1$ which violates our sensitivity condition of the process, hence we select $\gamma=0$, then Equation (26) becomes

$$
F(S, t, T)=\mathrm{e}^{L(t)(r-X(t)) \tau},
$$

Therefore, suppose that the rate of convenience yield follows the mean-reverting process then recall from Equation (17) that SDE;

$$
\mathrm{d} X(t)=\alpha(\mu-X(t)) \mathrm{d} t+\sigma \mathrm{d} L,
$$

where $\alpha>0$ is the speed of adjustment, $\mu$ is the long-run mean yield, and $\mathrm{d} L(t)$ is the increment to a standard Lévy process. However, from [13], the $\operatorname{cov}(\mathrm{d} L, \mathrm{~d} L)=\rho \mathrm{d} t$, where $\rho$ denotes the correlation coefficient. The variance of the change in the net marginal rate of convenience yield (volatility) is $\sigma^{2}$ representing the measure of jump sizes in the forward driven incomplete market.

Hence, we adopt [14] approach and construct a no-arbitrage portfolio that includes two futures contracts of different maturities and the spot commodity and this approach leads to the following differential equation for futures price;

$$
\begin{aligned}
& \frac{1}{2} F_{S S} S^{2} \sigma^{2}+\frac{1}{2} F_{X^{2}(t)} \sigma^{2}+F_{S X(t)} \rho \sigma^{2}+F_{S} S(r-X(t)) \\
& +F_{X(t)}(\alpha(\mu-X(t))-\lambda \sigma)-F_{\tau}=0
\end{aligned}
$$

with boundary condition

$$
F(S, X(t), 0)=S \text {. }
$$

Since convenience yield is non-traded, the differential Equation in (29) depends on investor risk preferences embedded in the market price of risk for convenience yield, $\lambda$. To obtain a solution, a version of the Feynman-Kac Theorem is involved and, for tractability, the market price of risk is assumed to be constant. This is equivalent to assuming in a general equilibrium framework that the representative investor has a logarithmic utility function. In this special case, the marginal utility of wealth is independent of wealth; and the market price of risk, which is given by the covariance of the change in the convenience yield with the rate of change in the marginal utility wealth, is constant.

Therefore, the Feynman-Kac solution for futures (forwards) price $F(t, T)$ is given by; 


$$
F(t, T)=\widehat{E}_{t}\left(S_{T}\right),
$$

where the expectation is taken with respect to the risk-neutral processes from Equation (17);

$$
\hat{\mathrm{d}} S=S(r-X(t)) \mathrm{d} t+S \sigma \mathrm{d} L
$$

and

$$
\hat{\mathrm{d}} X(t)=(\alpha(\mu-X(t))-\lambda \sigma) \mathrm{d} t+\sigma \mathrm{d} L .
$$

Then, we define the two-factor theoretical futures and forward prices depend on the current level of the spot price $S(t)$, the current level of the convenience yield, $X(t)$, time to maturity, the parameters of the joint process, and the price of a zero-coupon bond with maturity at time $T, P(t, T)$ based on similar approach by [13] such that suppose;

$$
Y(t)=\ln S-\int_{t}^{T} r(v) \mathrm{d} v .
$$

The standard Jump-diffusion for $Y(t)$ follows the transformation (34), Ito's lemma, and the risk-neutral diffusion for spot prices, and

$$
\hat{\mathrm{d}} Y(t)=\left(r-X(t)-\frac{1}{2} \sigma^{2}\right) \mathrm{d} t+\sigma \mathrm{d} L-\mathrm{d}\left(\int_{t}^{T} r(v) \mathrm{d} v\right) .
$$

Integrating (35) on both sides with respect to $T \geq t$;

$$
Y(t)=Y^{*}(t)-\int_{t}^{T} X(v) \mathrm{d} v-\frac{1}{2} \sigma^{2} \int_{t}^{T} \mathrm{~d} v+\sigma \int_{t}^{T} \mathrm{~d} L(v)
$$

however, from Equation (34) we take $Y^{*}(t)=\ln S$, then;

$$
Y(t)=\ln S(t)-\frac{1}{2} \sigma^{2}(T-t)-\int_{t}^{T} X(v) \mathrm{d} v+\sigma \int_{t}^{T} \mathrm{~d} L(v)
$$

since the effect of the interest rate in the drift of the spot process cancels out the effect of the interest rate discount factor. The forward solution is therefore given by

$$
F(t, T)=\frac{\widehat{E}_{t}\left[\mathrm{e}^{\ln S(t)-\frac{1}{2} \sigma^{2}(T-t)-\int_{t}^{T} X(v) \mathrm{d} v+\sigma \int_{t}^{T} \mathrm{~d} L(v)}\right]}{P(t, T)} .
$$

The solution for the forward price in Equation (37) is independent of the assumption of stochastic interest rates as long as $\sigma$ is independent of the spot interest rate, and $P(t, T)$ matches the market price of the zero-coupon bond with maturity at $T$ in a no-arbitrage interest model.

For the diffusion assumed for spot price, $\sigma$ is independent of $S$ and $r$, the distribution of $Y(t)$ is normal, and $\widehat{E}_{t}\left[\mathrm{e}^{Y(t)}\right]$ is the moment-generating function of a normal distribution. In this case, the solution for the forward price is

$$
F(t, T)=\frac{\mathrm{e}^{\widehat{E}_{t}[Y(t)]+\frac{1}{2} \operatorname{var}[Y(t)]}}{P(t, T)} .
$$


The expected value of $Y(t)$ is

$$
\widehat{E}_{t}[Y(t)]=\ln S(t)-\frac{1}{2} \sigma^{2}(T-t)-\int_{t}^{T} \widehat{E}_{t}[X(v)] \mathrm{d} v
$$

To solve the integral in (39), the solution for the risk-neutralized stochastic differential equation for convenience yield is used which is given by

$$
X(v)=\mu-\frac{\lambda \sigma}{\alpha}+\left(X(t)-\mu+\frac{\lambda \sigma}{\alpha}\right) \mathrm{e}^{-\alpha(v-t)}+\sigma \mathrm{e}^{-\alpha v} \int_{t}^{v} \mathrm{e}^{\alpha s} \mathrm{~d} L(s) .
$$

Substituting the expected value of Equation (40) into Equation (39) and integrating with respect to $\tau=T-t$ gives

$$
\widehat{E}_{t}[Y(T)]=\ln S(t)-\frac{1}{2} \sigma^{2} \tau-\left(\mu-\frac{\lambda \sigma}{\alpha}\right) \tau-\left(X(t)-\mu+\frac{\lambda \sigma}{\alpha}\right) H(\tau) .
$$

where

$$
E_{t}\left(\sigma \mathrm{e}^{-\alpha v} \int_{t}^{v} \mathrm{e}^{\alpha s} \mathrm{~d} L(s)\right)=0
$$

and

$$
H(T-t)=H(\tau)=\frac{1-\mathrm{e}^{-\alpha(\tau)}}{\alpha}
$$

The variance of $Y(t)$, Equation (36), is

$$
\operatorname{var}[Y(T)]=\operatorname{var}\left[\int_{t}^{T} X(v) \mathrm{d} v\right]+\sigma^{2} \tau-2 \operatorname{cov}\left[\int_{t}^{T} X(v) \mathrm{d} v, \int_{t}^{T} \sigma \mathrm{d} L(z)\right] .
$$

The variance and the covariance terms in Equation (42) are obtained by replacing $X(v)$ with the random part of the process (40), giving

$$
\begin{aligned}
\operatorname{var}[Y(T)]= & \operatorname{var}\left[\int_{t}^{T} \sigma \mathrm{e}^{-\alpha \nu} \int_{t}^{v} \mathrm{e}^{\alpha s} \mathrm{~d} L(s) \mathrm{d} v\right]+\sigma^{2} \tau \\
& -2 \operatorname{cov}\left[\int_{t}^{T} \sigma \mathrm{e}^{-\alpha \nu} \int_{t}^{v} \mathrm{e}^{\alpha s} \mathrm{~d} v, \int_{t}^{T} \sigma \mathrm{d} L(z)\right] .
\end{aligned}
$$

Solving for the variance and covariance in Equation (43) gives

$$
\operatorname{var}[Y(T)]=\left[-(H-\tau) \frac{\sigma^{2}}{\alpha^{2}}-\frac{\sigma^{2} H^{2}}{2 \alpha}\right]+\sigma^{2} \tau-\frac{2 \rho \sigma^{2}}{\alpha}(\tau-H) .
$$

The final result for $F(t, T)$ is obtained by using $\widehat{E}_{t}\left[\mathrm{e}^{Y(T)}\right]$, Equation (41), and $\operatorname{var}[Y(t)]$, Equation (44) in Equation (38) the forward solution is given by;

$$
F(S(t), X(t), t, T)=\frac{S(t) A(\tau) \mathrm{e}^{-H(\tau) X(t)}}{P(t, T)},
$$

where

$$
\begin{gathered}
A(\tau)=\exp \left[\frac{(H-\tau)\left(\alpha^{2} \mu-\alpha \lambda \sigma-\frac{\sigma}{2}+\rho \sigma^{2} \alpha\right)}{\alpha^{2}}-\frac{\sigma^{2} H^{2}}{4 \alpha}\right], \\
H=\frac{1-\mathrm{e}^{-\alpha \tau}}{\alpha}
\end{gathered}
$$


which gives the pricing framework for estimation of the energy forward dynamics modeled as a subordinated Hilbert space of linear functional.

\section{Main Results}

\subsection{Exploring the Forward Curve of Energy Commodity}

In this section, we select the West Texas Intermediate (WTI) Oil data for analysis and to evaluate the variation in forward rates in a given time interval. The data contains the daily prices of front month WTI oil price traded by NYMEX (New York Mercantile Exchange).

The front month WTI oil price is a futures contract with the shortest duration that could be purchased in the NYMEX market.

The WTI daily oil price is normalized in Figure 1 in other to refine the time series properties of the WTI oil data and the weight functions of the forward-style and the futures-style contract to enhance better pricing model for estimation of the forward prices. Between the first 50 monthly price observation, it is clear that noise exist in the data.

However, the spikes level is benchmarked using the properties Hilbert space linear functional of jump-reduction to reduce the price movement because the stochastic price return contains high volatility with massive jumps.

This is obvious in the daily change prices for the underlying asset as seen in Figure 2 and risk averse investors tend to drive the market with demand, the daily price change seems to favour the market share capitalization for a long period as seen in the forward curve.

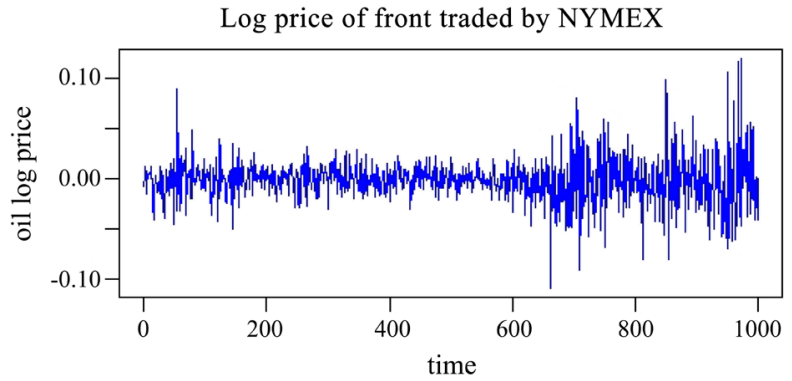

Figure 1. WTI crude oil price return with no-jump model.

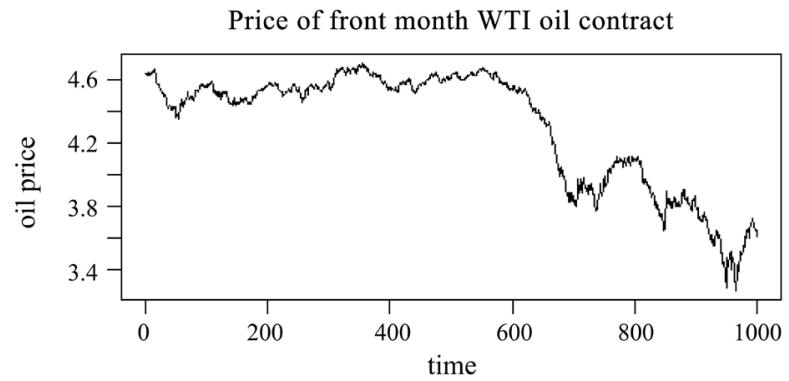

Figure 2. Forward rates forward curve of WTI crude oil price return with jump-diffusion. 
Furthermore, it follows from Equation (23) and as seen in Figure 2 that the dynamics of forward price curves can be represent as Hilbert space functional $H_{w}$ as a result of this assertion we accept that if $h(t)$ is an $H_{w}$-valued stochastic process, then $t \mapsto G_{\ell}^{w}(t)$ will be a stochastic process with values in $H_{w}$ as well.

As the subordinated process, the time change stochastic process $X(t)$ as shown in the forward curve in Figure 2 defines how the incomplete market trading options of WTI oil futures and forwards displays the highs and lows of the market situation.

Investors in the energy market are either willing to be risk neutral and trade for high profits or not but the energy forward pricing framework clearly favors the idea that with changing $\mu$ and $\sigma$ the forward rates $f(t, T)$ is bound to increase marginal yield over time $\tau=T-t$.

\subsection{Estimation of Forward Prices with and without Lévy Jump}

In order to avoid false detection of jumps in the forward curves pricing framework, we employed the following model for simulation;

$$
\mathrm{d} \log S(t)=\sigma(t) \mathrm{d} B(t)
$$

where $\sigma(t)$ is volatility, the drift component $\mu=0$ and $B(t)$ is a Wiener proces that follows a Poisson distribution $B_{t}-B_{0} \sim\left(\mu, \sigma^{2}\right) \sim((t, 0),(t, 0))$. In other to estimate the simulation model for a case of constant volatility verses stochastic volatility without Lévy jumps, we integrate both sides of Equation (46);

$$
S(\tau)=S(0) \mathrm{e}^{\sigma B(\tau)}
$$

With a constant volatility $\sigma=0.5$ and the standard Brownian motion following a Poisson distribution such that $B \in(0,1)$, then the simulated stock prices of the stochastic model is presented in Figure 3 with 1000 observations.

Figure 3 clearly indicates that the fluctuation in the stock prices without the presence of Lévy jumps is benchmarked by the volatility value of $\sigma=0.5$ upwards. However, we notice one spike in price of the stock which is absolute with a 0.05 level of significance and assigned to the variation in price dynamics of the model.

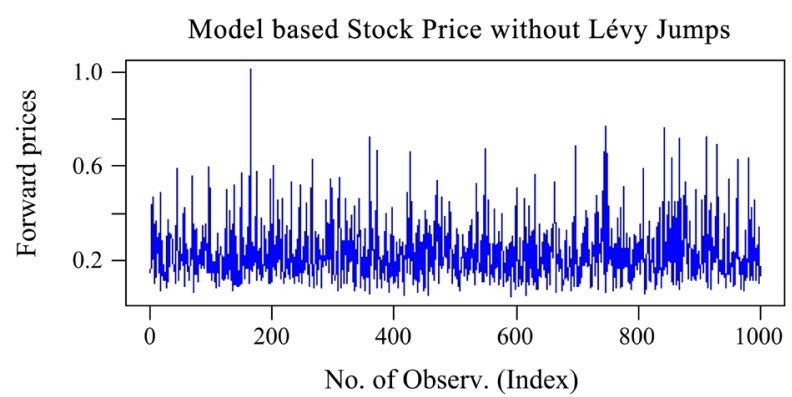

Figure 3. Model based stock prices without Lévy jumps. 
With a stochastic volatility $\sigma=1,2,3$ and the standard Brownian motion following a Poisson distribution such that $B \in(0,1)$, then the simulated stock prices of the stochastic model is presented in Figure 4 with 1000 observations.

With the big-jump and small-jump rules, Figure 5 clearly shows the detection and arrival of jumps from the small-jumps to big-jumps as the frequency of the observation increases with an initial volatility $\sigma=0.3$, given a sensitivity level $\gamma=0.0000>-1$. Although there exist a drift part $\alpha=0.05$, the number of jumps with constant volatility in all 300 observations is seen to be evenly distributed by both rules as can be seen in Table 1 .

The Log prices as described by Figure 5 and Figure 6 respectively shows that although there appears to be a varying changes in forward rates, with a slight change in volatility, the magnitude of price fluctuation in a stochastic volatility situation is the same with that of a constant volatility which further reinforces the impact of the presence of closed-gap normed spaces in the sequence of forward rates as introduce by the properties of the Banach space (special type of Hilbert space) (Table 2).

The forward curve without Lévy jumps as described in Figure 7 is estimated with the initial Lévy process $L(0)=0.525442$, the average stock price for WTI $S(0)=0.1163745$, and $\alpha=0.8857084$ is the drift component for which the price fluctuate with sensitivity and constant volatility of $\gamma=-0.8340501$, and $\sigma=0.3$ respectively where the jumps $\theta_{L, B}=1: 3, \theta_{L, S}=0.3$ are evaluated by the small-jump and big-jump rules accordingly with a convenient yield and correlation coefficient of the forward rates as $\lambda=0.2814486$ and $\rho=0.9591411$ respectively.

\section{Conclusion and Suggestions}

Representing energy forward and futures contracts as a time-changing stochastic process in a Hilbert-space of functions shows clearly, that an arbitrage-free forward price can be derived from the buy-and hold strategy in the energy market thereby enabling investors in the market willing to be salvage from the market uncertainties as well as Arrow-Debreu situations to execute a spot or forward contracts depending on the time and place the market becomes favorable.

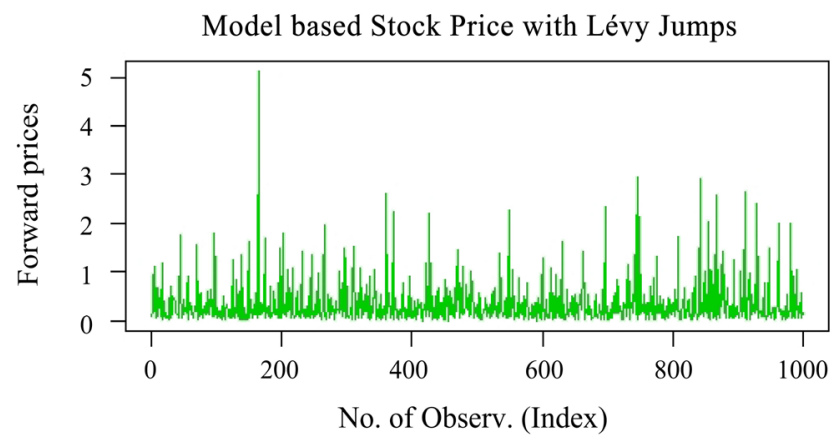

Figure 4. Model based stock prices without Lévy jumps. 


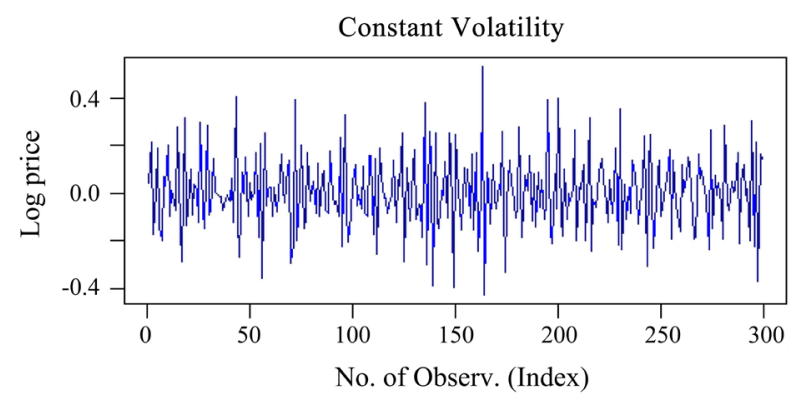

Figure 5. Simulated constant price volatility without jumps.

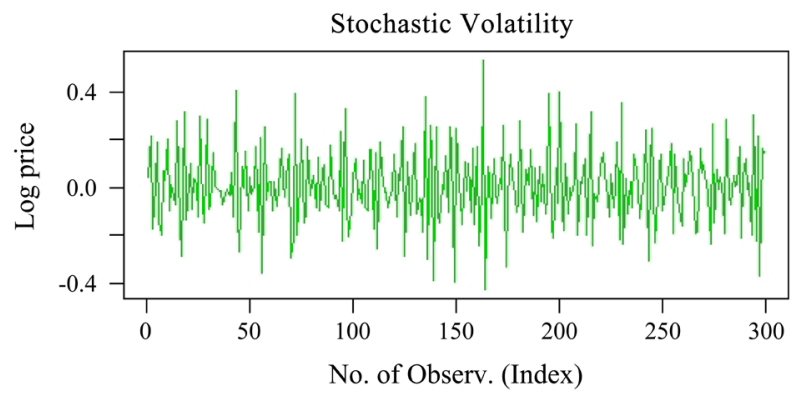

Figure 6. Simulated stochastic price volatility without Jumps.

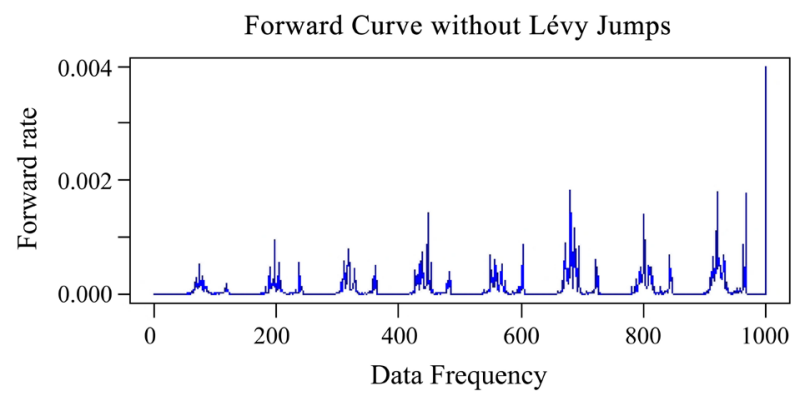

Figure 7. Estimated Forward curve for stock prices without Lévy jumps.

Table 1. Parameters of estimation for jump sizes with constant volatility.

\begin{tabular}{|c|c|c|c|c|}
\hline Time & Drift & Sensitivity & Volatility & Jump sizes \\
\hline$t$ & $\alpha$ & $\gamma$ & $\sigma$ & $L(t) \quad(\mathrm{S}=$ Small, $\mathrm{B}=\mathrm{Big})$ \\
\hline 1 & 0.9644 & 0.3762 & 0.9581 & $0.3534(\mathrm{~B})$ \\
\hline 2 & 0.7346 & 0.4773 & 0.9581 & $0.1575(\mathrm{~B})$ \\
\hline 3 & 0.7688 & 0.1321 & 0.9581 & $0.0484(\mathrm{~B})$ \\
\hline 4 & 0.5167 & 0.0968 & 0.9581 & $0.0206(\mathrm{~B})$ \\
\hline 5 & 0.0647 & 0.7966 & 0.9581 & $0.0082(\mathrm{~B})$ \\
\hline 6 & 0.6197 & 0.8012 & 0.9581 & $0.0041(\mathrm{~S})$ \\
\hline 7 & 0.2019 & 0.3994 & 0.9581 & $0.0023(\mathrm{~S})$ \\
\hline 8 & 0.5458 & 0.6292 & 0.9581 & $0.0008(\mathrm{~S})$ \\
\hline 9 & 0.4288 & 0.4571 & 0.9581 & $0.0003(\mathrm{~S})$ \\
\hline 10 & 0.2091 & 0.3334 & 0.9581 & $0.0001(\mathrm{~S})$ \\
\hline
\end{tabular}


Table 2. Parameters of estimation for jump sizes with stochastic volatility.

\begin{tabular}{ccccc}
\hline Time & Drift & Sensitivity & Volatility & Jump sizes \\
\hline$t$ & $\alpha$ & $\gamma$ & $\sigma$ & $L(t)$ \\
\hline 1 & 0.1569 & 0.1841 & 0.8959 & 0.3534 \\
2 & 0.7358 & 0.5716 & 0.2133 & 0.1575 \\
3 & 0.0542 & 0.6932 & 0.6018 & 0.0484 \\
4 & 0.9968 & 0.9028 & 0.7665 & 0.0206 \\
5 & 0.2605 & 0.4630 & 0.5737 & 0.0082 \\
6 & 0.1733 & 0.7254 & 0.7108 & 0.0041 \\
7 & 0.7742 & 0.2712 & 0.5577 & 0.0023 \\
8 & 0.7081 & 0.2887 & 0.3422 & 0.0008 \\
9 & 0.2679 & 0.0459 & 0.6776 & 0.0003 \\
10 & 0.7230 & 0.7954 & 0.8161 & 0.0001 \\
\hline
\end{tabular}

However, subsequent evaluation can take into consideration the impact of regime-shifts present in the time-changes as well as measure the sizes of such shifts and their corresponding impact to the price volatility.

\section{Primary Contribution}

- The multivariate subordinated processes are deduced as a moment generating function in order to bench mark the time-change process.

- The subordinated process in a continuous time is represented as a stochastic integral as Hilbert space linear functional.

- The energy forward is represented while incorporating the completeness properties of Banach space (a special type of Hilbert space) for an incomplete energy market.

- The pricing framework for energy forward contracts is used to evaluate the daily change price of an underlying energy contracts.

\section{Acknowledgements}

Our sincere thanks to the Pan African University Institute for Basic Science and Technology, Kenya for their support, and special thanks to Dr. Jane Aduda Akinyi for moral support guide throughout the development of this paper.

\section{Conflicts of Interest}

The authors declare no conflicts of interest regarding the publication of this paper.

\section{References}

[1] Barndorff-Nielsen, O.E., Benth, F.E. and Veraart, A.E. (2013) Modelling Energy Spot Prices by Volatility Modulated Lévy-Driven Volterra Processes. Bernoulli, 19, 803-845. https://doi.org/10.3150/12-BEJ476 
[2] Benth, F.E., Benth, J.S. and Koekebakker, S. (2008) Stochastic Modelling of Electricity and Related Markets. World Scientific, 11, 352. https://doi.org/10.1142/6811

[3] Benth, F.E. and Kruhner, P. (2015) Derivatives Pricing in Energy Markets: an Infinite-Dimensional Approach. SIAM Journal on Financial Mathematics, 6, 825-869. https://doi.org/10.1137/15100268X

[4] Benth, F.E. and Zdanowicz, H. (2016) Pricing and Hedging of Energy Spread Options and Volatility Modulated Volterra Processes. International Journal of Theoretical and Applied Finance, 19, No. 1. https://doi.org/10.1142/S0219024916500023

[5] Benth, F.S. and Benth, J.S. (2012) Modeling and Pricing in Financial Markets for Weather Derivatives. Technical Report, World Scientific, Singapore. https://doi.org/10.1142/8457

[6] Benth, F.E. and Krühner, P. (2012) Subordination of Hilbert Space Valued Lévy Processes. arXiv preprint arXiv:1211.6266.

[7] Clewlow, L. and Strickland, C. (2000) Energy Derivatives: Pricing and Risk Management. Lacima Publications, London.

[8] Filipovic, D. (2001) Consistency Problems for Heath-Jarrow-Morton Interest Rate Models. Springer Science \& Business Media. https://doi.org/10.1007/b76888

[9] Hilliard, J.E. and Reis, J. (1998) Valuation of Commodity Futures and Options under Stochastic Convenience Yields, Interest Rates, and Jump Diffusions in the Spot. Journal of Financial and Quantitative Analysis, 33, 61-86.

https://doi.org/10.2307/2331378

[10] Hull, J. and White, A. (1987) The Pricing of Options on Assets with Stochastic Volatilities. The Journal of Finance, 42, 281-300. https://doi.org/10.1111/j.1540-6261.1987.tb02568.x

[11] Kallsen, J. and Kruhner, P. (2014) On a Heath-Jarrow-Morton Approach for Stock Options. Finance and Statistics, 19, 583-615. https://doi.org/10.1007/s00780-015-0263-1

[12] Kilian, L. and Murphy, D. (2013) The Role of Inventories and Speculative Trading in the Global Market for Crude Oil. Journal of Applied Econometrics, 29, 454-478. https://doi.org/10.1002/jae.2322

[13] Monroe, I. (1972) On Embedding Right Continuous Martingales in Brownian Motion. Annals of Mathematical Statistics, 43, 1293-1311.

[14] Peszat, S. and Zabczyk, J. (2007) Stochastic Partial Differential Equations with Lévy Noise. Cambridge University Press, Cambridge. https://doi.org/10.1017/CBO9780511721373 